\title{
The electroencephalogram after resuscitation of cardiocirculatory arrest
}

\author{
HELIO LEMMI, CHARLES H. HUBBERT, AND AMIN A. FARIS \\ From the Neurophysiology Laboratory and Departments of Neurology and Medicine \\ of the Baptist Memorial Hospital and University of Tennessee College of Medicine, \\ Memphis, Tennessee, U.S.A.
}

SUMMARY Fifty-two EEGs of 31 patients were studied after resuscitation from cardiac arrest. Examples of patients with normal or mildly abnormal (category I) and severely abnormal (category II) EEGs are presented. All patients in category II died. In the patients of category I, EEGs showing no improvement or worsening indicated a fatal prognosis and possibly reflected deteriorating cardiac function caused by the basic disease process.

Recent studies have made reference to the prognosis of patients who were resuscitated after cardiocirculatory arrest, and some used the electroencephalogram (EEG) as at least one of the criteria for prognosis (Hockaday et al., 1965; Binnie et al., 1970; Kurtz et al., 1970). Pampiglione and Harden (1968) published a study of the EEG reports of the previous 10 years. In view of the opportunity afforded at the Baptist Memorial Hospital of Memphis, Tennessee, which has a very active resuscitation service, we have been able to study a group of similar but adult patients in light of Pampiglione and Harden's findings.

\section{METHODS}

Over a 12 month period, 31 patients were selected for this study. Fifty-two electroencephalograms were obtained. The EEGs were recorded on Grass Model VI and Beckman Model CE electroencephalograph machines using standard international electrode placements of surface silver-silver chloride electrodes applied with salt jelly and secured with collodion. Referential and bipolar montages were recorded. Although judicious use of filters and amplifications was made, by and large, as in samples of Figs 1, 2, and 3, high frequency filters were set at $70 \mathrm{~Hz}$ and time constant at 0.3 seconds.

The observation of each patient included the type of cardiac arrest which was substantiated by the electrocardiogram, the type of primary disease pro- cess, and the neurological and physical clinical course. Twenty-two patients were men and nine were women. They varied in age from 18 to 83 years. The criteria for inclusion in this study were: (1) the patient must have had cardiocirculatory arrest requiring one or more therapeutic methods of resuscitation such as a strong blow to the thorax, external

TABLE 1

INCIDENCE AND AGE RANGE IN PRIMARY DISEASE

\begin{tabular}{lcr}
\hline Primary disease & $\begin{array}{c}\text { Age range } \\
(y r)\end{array}$ & $\begin{array}{c}\text { Cases } \\
(\text { no. })\end{array}$ \\
\hline Atherosclerotic heart disease (ASHD) with & & \\
$\quad$ myocardial infarction & $43-75$ & 20 \\
ASHD alone & $42-81$ & 3 \\
Hypertensive cardiovascular disease & $52-61$ & 2 \\
Systemic lupus erythematosus & 57 & 1 \\
Pulmonary embolism & 18 & 1 \\
Glomerulonephritis and chronic renal failure & 25 & 1 \\
Postoperative state for perforated peptic ulcer & 68 & 1 \\
Acute haemorrhagic pneumonitis & 73 & 1 \\
Cardiomyopathy & 63 & 31 \\
\hline Total & &
\end{tabular}

cardiac massage, artificial respiration, drugs, defibrillation, and cardiac pace-maker; (2) an electrocardiogram showing ventricular tachycardia, ventricular fibrillation, asystole or heart block. Cases of arterial hypotension, bradycardia, respiratory insufficiency or arrest alone, trauma, neoplasia, infections, and intoxications were excluded from the study. 
The patients were resuscitated by physicians proficient in this procedure and who were either at the bedside or arrived to aid the patients within two minutes (most authorities feel that the critical time for resuscitating such individuals is up to four or five minutes). In a few cases the nursing staff had already initiated some of the resuscitative procedures.

Table 1 shows the incidence of the type of primary disease in our patients and their age range. The predominant incidence of arteriosclerosis with myocardial infarction is clear.

TABLE 2

EEG CLASSIFICATION

\footnotetext{
Category I

a-Normal

b-Alpha rhythm with mixed slow activity of theta and delta ranges

Category II

a-Diffuse slow activity - theta and delta

b-Severe cortical suppression; occasional low voltage fast activity c-Burst-suppression

d-Low voltage, slow waves and also sharp or spike waves
}

TABLE 3

DEATH IN RELATION TO CATEGORY

\begin{tabular}{ccc}
\hline Category & $\begin{array}{c}\text { Patients } \\
\text { (no.) }\end{array}$ & Deaths \\
\hline Ia & 4 & 1 \\
Ib & 17 & 7 \\
IIa & 1 & All \\
IIb & 2 & All \\
IIc & 3 & All \\
IId & 4 & All \\
\hline
\end{tabular}

The EEGs were obtained as soon as it became possible to record at the bedside without interfering with other examination and treatment directed toward the circulatory emergency. Most patients had the EEG recorded between six and 24 hours after cardiac arrest except four patients who had the first EEG recorded between 36 and 72 hours of the resuscitation. As pointed out by Kurtz et al. (1970) EEGs after cardiac arrest done within a period from 15 to 24 hours are more significant for the prediction of the clinical evolution than a study obtained in the first few hours after resuscitation. It is felt that the initial EEG obtained within 24 hours in most of our cases fitted this observation, since this timing resulted in a more stable EEG for the purposes of prediction of outcome, although Pampiglione and Harden's (1968) timing for optimum prognostic value of the EEG in children was somewhat less.

The EEG records were subsequently analysed and divided into two categories: I-those still containing alpha rhythm (Fig. 1) and II-those which did not contain alpha rhythm (Fig. 2). Table 2 shows such classification in more detail according to the findings detected by the first EEG obtained in each patient. Table 3 shows the number of patients and survivors in each category. The separation of the patients in category I with a normal EEG (Ia) from those containing predominant alpha rhythm but with intermixed slowing (Ib) is of practical importance (vide infra). Our classification is otherwise similar to that used by Pampiglione and Harden (1968).

FIG. 1. EEGs of a patient in category $I$, group $b$. A: sample at 16 hours after arrest. Note mild intermixed slowing and poor regulation. B: the follow-up seven days later is normal. 


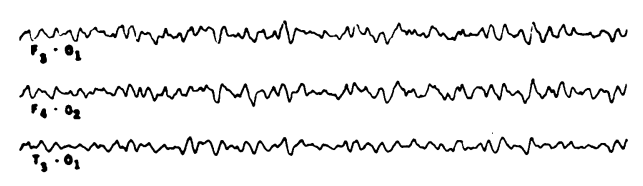

A

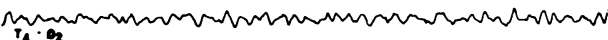

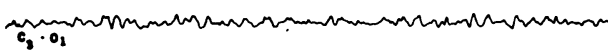

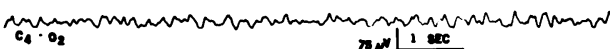

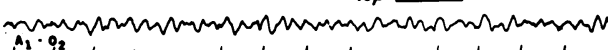

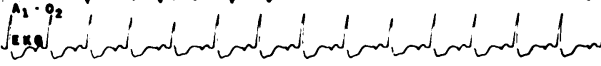

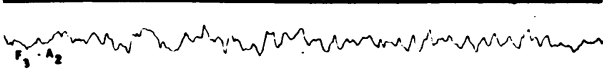

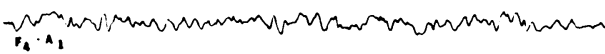

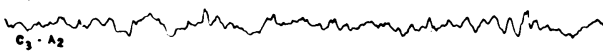

\section{IIIa}

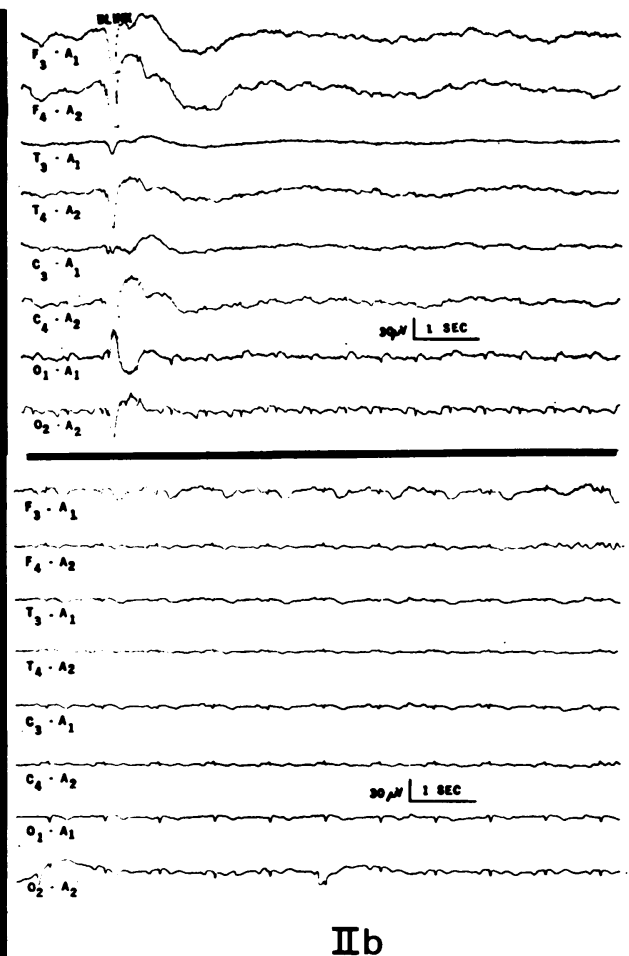

A

B

\section{CASE ILLUSTRATIONS}

CATEGORY Ia A 42 year old man with arteriosclerotic heart disease (angina) developed ventricular fibrillation during coronary angiography. The patient was immediately defibrillated and clinically did not seem to suffer any physical or, in particular, neurological damage. He did not lose consciousness. The coronary arteriogram showed obstruction of the right coronary artery. An EEG obtained seven hours after the circulatory arrest was normal. The patient was discharged in five days. A few months later, he underwent a successful coronary by-pass grafting and has continued to do well.

CATEGORY Ib A 25 year old woman with compensated renal insufficiency secondary to chronic glomerulonephritis suddenly developed complete heart block which resulted in a ventricular rate of 30 per minute. There was no loss of consciousness. The resuscitative effort was successful and the patient recovered without apparent neurological sequelae. Figure 1 (Ib) shows an example of the EEG obtained in 16 hours (A) and seven days later (B). The later record shows clear improvement. She was discharged home in 19 days where she continues to do satisfactorily.

CATEGORY IIa A 67 year old man with a history of arteriosclerotic heart disease and several previous myocardial infarctions and pulmonary oedema was admitted to the Emergency Room and found to be without signs of life. An electrocardiogram showed ventricular fibrillation. Cardiac resuscitation was successful, but the patient remained in a coma and died seven days later. His EEG at 24 hours showed diffuse slow activity of theta and delta frequencies without definite focalization (Fig. 2, IIa-A). A follow up EEG four days after the arrest (B) shows even slower activity, again of a generalized nature.

CATEGORY IIb A 48 year old man had had a myocardial infarction and was being cared for in the Cardiac Unit when he suddenly developed cardiac arrest (asystole). Although he was promptly resuscitated as to cardiac function he did not recover 

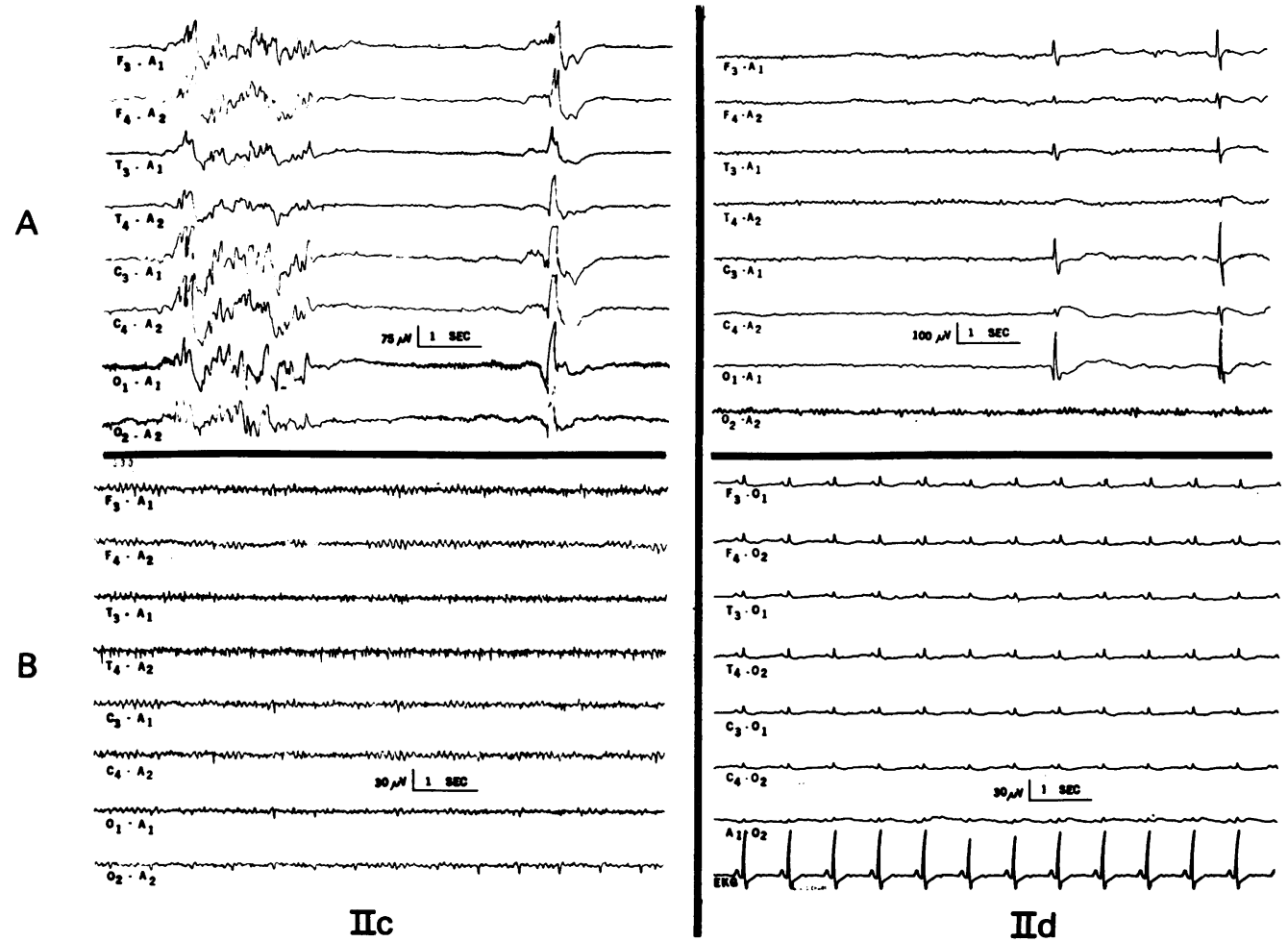

FIG. 3. Category IIc-A: sample obtained 13 hours after arrest showing burst-suppression activity. B: three days later shows 'alphoid' rhythm of very low voltage. IId-A: nine hours after arrest the EEG shows low voltage slow activity as well as isolated spikes. B: seven days later shows severe cortical suppression.

consciousness and died in 15 days. The first EEG obtained 24 hours after arrest showed suppression of voltage with very slow basic rhythm throughout. The patient was in a coma vigil, thus explaining the occasional blink artefact seen in Fig. 2 (IIb-A). The follow-up EEG six days later (B) showed no changes.

CATEGORY IIC A 60 year old woman with myocardial infarction and subsequent cardiac arrest (asystole) went into coma and continued in that state until death six days later. Approximately 15 hours after cardiac resuscitation she developed convulsions and shortly thereafter demonstrated a decerebrate state. The EEG obtained 13 hours after the cardiac arrest (Fig. 3, IIc-A) shows alternating bursts-suppression activity. The follow-up EEG (B) obtained three days after the original study shows a monorhythmic activity in the alpha range while the patient remained in a coma.

CATEGORY IId A 43 year old woman known to be hypertensive suffered cardiac arrest (asystole) as she reached the Emergency Room after having suddenly lost consciousness at home. Although the resuscitative effort was successful for cardiac function, the patient remained in a coma. In a few hours she developed a decerebrate state with intermittent generalized convulsions. An EEG obtained nine hours after cardiac arrest showed low voltage slow activity in the delta range throughout, intermixed with more rapid rhythms and isolated high voltage spike discharges which were slightly lateralized to the left (Fig. 2, IId-A). A follow-up sample seven days later (B) demonstrated only severe cortical suppression. The patient died one day later without further clinical changes. Necropsy revealed myocardial infarction. Study of the brain was incomplete since postmortem autolytic changes masked other possible findings.

Follow-up of the 13 survivors, 12 to 24 months after arrest, showed 12 patients alive and doing well from the neurological standpoint. One patient died one year after cardiac arrest of a similar episode from which she could not be resuscitated. 


\section{DISCUSSION}

The cases illustrated above are representative of all in not providing an explanation for the cardiac arrest. In some a cerebral pathological process may have anticipated the cardiac arrest rather than being secondary. Nevertheless, since we chose to divide the cases into two major categories of those retaining alpha rhythm and those without, it was apparent that regardless of the pathology in the brain preceding cardiac arrest or vice versa, some prognostic determination could be made both clinically and electroencephalographically. It became quite obvious that the patients who were unconscious from the onset of cardiac arrest and who remained so until the time they had their first EEG (category II) had no chance of survival. Therefore, the type of EEG abnormality in itself was not helpful in predicting the chances of survival for this group. Conversely, the group who retained the alpha activity and who were only briefly unconscious or not unconscious at all, and who were alert at the time of the first EEG, had a much better chance of survival, particularly if their EEG was entirely normal. Of four such patients constituting our category Ia, all but one survived. The one death occurred three weeks later from another cardiac arrest. In category Ib, 17 patients were studied, and, although they preserved some alpha activity, their EEG records were not normal because they contained intermixed slowing indicating mild diffuse cerebral dysfunction interpreted as probably secondary to anoxia. Seven patients in this group died. Their follow-up records a week later showed no improvement or worsening.

Our findings in general match those of Pampiglione and Harden (1968) who, dividing their cases into four groups, found that all children showing well-preserved EEG features appropriate to their age survived while those in the three groups who had either diffusely slow flat patterns with bursts of spikes or slow waves, or iso-electric EEGs died, with one exception. Their only survivor lived for six years in a decerebrate state. Hockaday et al. (1965) divided their patients into five groups. The first two groups had preserved alpha rhythm and the latter three had essentially no alpha rhythm. The majority of their patients in the more severe categories of EEG dysfunction died without regaining consciousness. Only one of these cases survived showing improvement in the EEG. This patient however, remained with dementia, clonus, and spasticity. Binnie et al. (1970) reported 44 patients with cardiac arrest of whom 23 survived.

These authors discussed the EEG findings of prognostic value and specifically cite a patient in deep coma and unresponsive to stimuli whose EEG showed low amplitude rhythmical activity in the alpha range. Ordinarily, the retention of alpha rhythm would suggest a good prognosis, but in their comatose patient the prognosis was predictably fatal. Our case example of category IIc appears similar to this description. Such patterns in the comatose patient appear to reflect brain-stem pathology. This is supported by the report of Otomo (1966) of three patients who had had acute cerebral vascular disorders involving the brain-stem. They were comatose but did not show the high voltage slow wave activity in the EEG which is usually associated with such deep coma. Their EEG showed predominant rhythm of alpha frequency and low voltage fast activity, with relatively less higher voltage slow activity a few hours after the onset of the coma. Little intracerebral pathology was found on necropsy. Unfortunately, we were unable to obtain a necropsy in our case.

As the data obtained in our study compare well with those above, and in general match the findings in children with the same problem, it becomes evident that the EEG can be of practical importance in the general assessment of prognosis in a patient with cardiac arrest.

We are generally at a loss to explain why the electrical activity of the brain, particularly in those cases of patients who were alert and still preserving alpha activity but yet who later died, has anything to do with the grave prognosis of impending death. A probable explanation has been advanced by Schneider (1961) who pointed out that circulatory arrest in itself produces no cerebral damage for at least eight to 10 minutes. However, many other organs, including the heart itself, cannot endure ischaemia for a period longer than about four minutes. Generally one accepts it as a fact that, if the heart can return to functioning properly, this is an indication that the cerebral circulation has also returned to 
normal, and therefore no damage should be expected. This is not always the case. Cardiac and cerebral ischaemia, secondary to the cardiac arrest, superimposed on the basic cardiac disease present before arrest has a further deleterious effect on the heart itself even though resuscitation seemingly restores it towards previous levels of function. It is likely that, as cardiac insufficiency continues or is aggravated it adds to the initial cerebral insult, thus reflecting increased dysfunction in the EEG, which worsens. Furthermore, the cardiac insufficiency continues to affect the function of other vital organs as well, all of which contribute to the patient's demise. Thus, the cerebral abnormality as described by the EEG must indirectly reflect the general condition of the individual who has suffered a previous cardiac arrest and possibly, in particular, reflects cardiac status. The findings assume prognostic value especially in those cases of category Ib in which the EEG shows worsening in contrast with those in which the follow-up EEG shows improvement. The patients in this group who died showed continuous evidence of cardiac dysfunction and their deaths were attributed to a cardiac cause.
We wish to thank Dr. Pervis Milnor, Jr., Chief of Medicine, Baptist Memorial Høspital and his staff, particularly of the Resuscitating Team for allowing us to study their cases. We thank Mr. Joseph Belluomini, Mrs. G. K. Ballard, and Mrs. C. L. Rutherford for their technical and secretarial assistance.

\section{REFERENCES}

Binnie, C. D., Prior, P. F., Lloyd, D. S. L., Scott, D. F., and Margerison, J. H. (1970). Electroencephalographic prediction of fatal anoxic brain damage after resuscitation from cardiac arrest, British Medical Journal, 4, 265-268.

Hockaday, J. M., Potts, F., Epstein, E., Bonazzi, A., and Schwab, R. S. (1965). Electroencephalographic changes in acute cerebral anoxia from cardiac or respiratory arrest. Electroencephalography and Clinical Neurophysiology, 18, 575-586.

Kurtz, D., Cornette, M., Tempe, J. D., and Mantz, J. M. (1970). Prognostic value of the EEG following reversible cardiac arrest. From 90 cases. (Abstract.) Electroencephalography and Clinical Neurophysiology, 29, 530-531.

Otomo, E. (1966). Beta wave activity in the electroencephalogram in cases of coma due to acute brain-stem lesions. Journal of Neurology, Neurosurgery, and Psychiatry, 29, 383-390.

Pampiglione, G., and Harden, A. (1968). Resuscitation afte? cardiocirculatory arrest. Lancet, 1, 1261-1265.

Schneider, M. (1961). Survival and revival of the brain in anoxia and ischemia. In Cerebral Anoxia and the Electro encephalogram, pp. 134-143. Edited by H. Gastaut and J. S. Meyer. Thomas: Springfield, Ill. 